\title{
Engineering Atomically Sharp Potential Steps and Band Alignment at Solid Interfaces using 2D Janus Layers
}

Riis-Jensen, Anders C.; Manti, Simone; Thygesen, Kristian S.

Published in:

Journal of Physical Chemistry C

Link to article, DOI:

10.1021/acs.jpcc.0c01286

Publication date:

2020

Document Version

Early version, also known as pre-print

Link back to DTU Orbit

Citation (APA):

Riis-Jensen, A. C., Manti, S., \& Thygesen, K. S. (2020). Engineering Atomically Sharp Potential Steps and Band Alignment at Solid Interfaces using 2D Janus Layers. Journal of Physical Chemistry C, 124(17), 9572-9580. https://doi.org/10.1021/acs.jpcc.0c01286

\section{General rights}

Copyright and moral rights for the publications made accessible in the public portal are retained by the authors and/or other copyright owners and it is a condition of accessing publications that users recognise and abide by the legal requirements associated with these rights.

- Users may download and print one copy of any publication from the public portal for the purpose of private study or research.

- You may not further distribute the material or use it for any profit-making activity or commercial gain

- You may freely distribute the URL identifying the publication in the public portal 


\title{
Predictive Engineering of Atomically Sharp Potential Steps and Band Alignment at Solid Interfaces
}

\author{
Anders C. Riis-Jensen, ${ }^{\dagger}$ Simone Manti, ${ }^{\dagger}$ and Kristian S. Thygesen ${ }^{*, \dagger, \ddagger}$ \\ $\dagger C A M D$, Department of Physics, Technical University of Denmark, DK - 2800 Kongens \\ Lyngby, Denmark \\ $\ddagger$ Center for Nanostructured Graphene (CNG), Department of Physics, Technical University \\ of Denmark, DK - 2800 Kongens Lyngby, Denmark \\ E-mail: thygesen@fysik.dtu.dk
}




\begin{abstract}
The possibility of stacking two-dimensional (2D) materials into van der Waals (vdW) heterostructures has recently created new opportunities for band structure engineering at the atomic level. However, despite of the weak vdW interaction, controlling the electrostatic potential governing the band line-up at the 2D interfaces is still posing a significant challenge. Here we demonstrate that 2D Janus monolayers possessing an intrinsic out-of-plane dipole moment, can be used to control the band alignment at semiconductor-semiconductor and metal-semiconductor interfaces in a highly predictive manner. Using density functional theory (DFT), we calculate the band structure of a wide range of different vdWs interfaces. We find that upon insertion of a Janus structure, band line-ups and Schottky-barriers can be controlled to high accuracy. A main result of this work, is that the out-of-plane dipole moment of the Janus structure changes little upon insertion in the interface. As a consequence the effect on the electrostatic potential at the interface can be predicted from the properties of the freestanding Janus structure. In addition to this, we predict 47 stable Janus monolayers, covering a wide range of dipole moments and band edge positions, thus providing a comprehensive library of $2 \mathrm{D}$ building blocks for manipulating band alignment at interfaces.
\end{abstract}

\title{
Introduction
}

More than a decade after the discovery of graphene, ${ }^{1}$ the class of atomically thin twodimensional (2D) crystals remains one of the hottest topics in physics. An important reason is that such materials open new possibilities for studying and manipulating electronic quantum states directly at the atomic length scale. By stacking different 2D layers into van der Waals (vdW) heterostructures it is, at least in principle, possible to design the energy landscape of the electrons, i.e. the band structure, with a precision far beyond what is possible with conventional epitaxial growth. ${ }^{2}$ In this paper we introduce the idea of using 2D Janus monolayers to control the electrostatic potential and band line-up at vdW interfaces.

Many solid state devices, including semiconductor (SC) lasers,,${ }^{3-5}$ solar cells, ${ }^{6,7}$ and tran- 
sistors ${ }^{8}$ rely on heterostructure materials with electronic energy levels carefully aligned across their interfaces. This makes the interfacial band lineup problem one of the most critical challenges for the semiconductor industry. Quite generally, the band alignment at SC heterojunctions can be categorized into three types according to the relative position of valence and conduction bands on the two sides of the interface. In figure 1 we show examples of type-I and type-II band alignments. With conventional SC technology and materials it is very difficult, if not impossible, to change the type of band alignment at a given heterojunction without changing the composition or composition of at least one of the SC materials, e.g. by strain, doping, or alloying. It is exceedingly difficult, by means of conventional techniques employed in the semiconductor industry, to change the band alignment at an interface without changing the composition or structure of the material(s) in the vicinity of the interface. This clearly implies a risk of degrading the materials properties e.g. the carrier mobility or -lifetime, etc. A key result of this paper is that the band alignment can be controlled elegantly and with high precision in vdW heterojunctions without affecting the materials structure or composition.

For metal/SC interfaces, the single most critical parameter used to characterize the band alignment is the Schottky barrier (SB) height, i.e. the distance between the metal Fermi level and the nearest band edge of the $\mathrm{SC}$ (valence band or conduction band). Apart from the position of the bands in the intrinsic materials, i.e. the work function of the metal and the electron affinity and ionization potential of the SC, a number of effects influence the SB including Pauli repulsion and metal induced gap states (MIGS). ${ }^{9-13}$ Such effects lead to Fermi level pinning ${ }^{10,14-16,16-22}$ (FLP) and deviations from the Anderson rule ${ }^{23}$ which makes it difficult to control (in practice minimize) the SB. This is further complicated by the unpredictable effect of the chemical interactions at the interface. One specific and important motivation for developing means to control the SB at metal-2D semiconductor interfaces comes from the huge interest in atomically thin field effect transistor based on transition metal dichalcogenides (TMDs). For such devices the SB at the source/drain contacts remains 
a performance limiting factor. ${ }^{24-33}$

Due to the presence of MIGS it has been proven ineffective to dope SC/metals structures to lower the SB. This has previously been explained by the fact that while originating on the metal, the states are still localized deeply into the $\mathrm{SC},{ }^{34}$ effectively neutralizing the effect of doping. Approaches to overcome this includes inserting a layer of boron nitride between the metal and the $\mathrm{SC}^{22,35}$ or different oxide layers,${ }^{36-39}$ however the exact effect of all of the above mentioned mechanisms is extremely difficult to predict.

In this work we propose an alternative method to control and adjust the band lineup at solid interfaces in a highly predictable manner. By sandwiching a 2D material with a finite out-of-plane dipole moment, i.e. a Janus monolayer, between two materials, it is possible to introduce an atomically sharp potential step across the interface. For SC/SC interfaces this opens the possibility of controlling the band line-up and even changing the type of band alignment, while for metal/SC interfaces the SB can essentially be made to vanish. Importantly we find that the dipole moment of the Janus layer does not change significantly upon insertion in the heterostructure, meaning that the resulting shift in the band offset can be predicted to high accuracy from the properties of the isolated Janus monolayer, for systems with small interlayer charge transfer. Beyond pure vdW-bonded structures we find that the intrinsic dipole moment is unaltered, even when placed on conventional bulk metal surfaces. Using high-throughput DFT computations we further predict 47 new Janus monolayers that we find to be stable, have a finite band gap, and a finite out-of-plane dipole moment. The induced shift in electrostatic potential achievable by these novel 2D Janus monolayerss range from close to zero to about $2 \mathrm{eV}$, showing the high degree of band offset tunability offered by the proposed concept.

\section{Computational details}

All calculations were perfomed with the GPAW code. ${ }^{40}$ Monolayer structures were relaxed using the PBE functional ${ }^{41}$ on a Monkhorst-Pack $k$-point grid $^{42}$ with a $k$-point density of 6.0 
and a plane-wave cut-off of $800 \mathrm{eV}$. The unit cells had $15 \AA$ of vacuum in the perpendicular direction and a Fermi-smearing of $0.05 \mathrm{eV}$ was used. The band structure and the size of the dipole moment were calculated using a $k$-point density of 12.0. Spin-orbit coupling was not included. For the multilayer structures, the interlayer binding distances were determined using the BEEF-vdW functional. ${ }^{43}$ All structures were relaxed until the maximum force on any atom was below $0.01 \mathrm{eV} / \AA$ and the maximum stress on the unit cell was $0.002 \mathrm{eV} / \AA^{3}$. Since the layers in the heterostructures in general will have incommensurable lattices, it is necessary to use larger supercells and strain one or both materials slightly. Table 1 gives a summary of the supercell size and strain applied to each layer in each heterostructure. For H-phase/H-phase interfaces AB stacking was used while for H-phase/T-phase interfaces AA stacking was used, i.e. the metal atoms were stacked on top of each other. These stacking configurations were found to be energetically most stable. To judge the stability (dynamic and thermodynamic) of the new Janus monolayers resulting from the high-throughput study, we followed the criteria of the Computational 2D Materials Database. ${ }^{44}$

\section{Results}

Recently both MoSSe ${ }^{45,46}$ and BiTeI ${ }^{47}$ have been realized in monolayer form experimentally. These structures posses an out-of-plane dipole moment created by the difference in electronegativity of the $\mathrm{S}$ and $\mathrm{Se}$ (I and Te) atoms, ${ }^{48}$ which are located on different sides of the central metal layer, see figure 2 .

Such structures with broken mirror symmetry and a finite out-of-plane dipole moment are known as Janus monolayers. In figure 1 we illustrate the concept of band lineup control at vdW heterojunction interfaces. On the left is shown an example of a bilayer consisting of two (possibly) different semi-conducting 2D monolayers having a type-I band alignment. On the right is shown the same two monolayers, with a Janus monolayer sandwiched in-between. By choosing a Janus monolayer with an appropriate size of the out-of-plane dipole moment, it is possible to shift, for instance, a type-I band alignment into a type-II band alignment as 
Table 1: Computational details about the supercells used for the heterostructure calculations including cell size, strain, and smallest interlayer distance $d$.

\begin{tabular}{|c|c|c|c|}
\hline Structure & Cell size & Strain [\%] & $d[\AA]$ \\
\hline Graphene/H-MoSSe/hBN & $\begin{array}{c}\text { Graphene: } 4 \times 4 \\
\text { H-MoSSe: } 3 \times 3 \\
\text { hBN: } 4 \times 4\end{array}$ & $\begin{array}{c}1.62 \\
-0.46 \\
3.31\end{array}$ & $3.82 / 3.69$ \\
\hline T-MoS $2 / \mathrm{H}-\mathrm{MoSSe} / \mathrm{H}-\mathrm{MoS}_{2}$ & $\begin{array}{c}\text { T-MoS }: 1 \times 1 \\
\text { H-MoSSe: } 1 \times 1 \\
\text { H-MoS } 2: 1 \times 1\end{array}$ & $\begin{array}{c}0 \\
-2.06 \\
0\end{array}$ & $3.51 / 3.67$ \\
\hline H-MoS $2 / \mathrm{H}-\mathrm{MoSSe} / \mathrm{H}-\mathrm{MoS}_{2}$ & $\begin{array}{c}\text { H-MoS }: 1 \times 1 \\
\text { H-MoSSe: } 1 \times 1\end{array}$ & $\begin{array}{c}0 \\
-2.06\end{array}$ & $3.54 / 3.63$ \\
\hline $\mathrm{H}-\mathrm{WS}_{2} / \mathrm{H}-\mathrm{MoSSe} / \mathrm{H}-\mathrm{WS}_{2}$ & $\begin{array}{c}\text { H-WS } \\
\text { H-MoSSe: } 1 \times 1 \\
\text {-Mo } 1\end{array}$ & $\begin{array}{c}0 \\
-2.06\end{array}$ & $3.52 / 3.66$ \\
\hline $\mathrm{H}-\mathrm{MoS}_{2} / \mathrm{H}-\mathrm{CrSSe} / \mathrm{H}-\mathrm{MoS}_{2}$ & $\begin{array}{l}\text { H-MoS }: 1 \times 1 \\
\text { H-CrSSe: } 1 \times 1\end{array}$ & $\begin{array}{c}0 \\
1.76 \\
\end{array}$ & $3.61 / 3.75$ \\
\hline $\mathrm{H}-\mathrm{MoSe}_{2} / \mathrm{H}-\mathrm{MoSTe} / \mathrm{H}-\mathrm{MoSe}_{2}$ & $\begin{array}{l}\text { H-MoSe }: 1 \times 1 \\
\text { H-MoSTe: } 1 \times 1\end{array}$ & $\begin{array}{c}0 \\
<0.01 \\
\end{array}$ & $3.61 / 3.74$ \\
\hline H-MoSe $2 / \mathrm{H}-\mathrm{TaSSe} / \mathrm{H}-\mathrm{MoSe}_{2}$ & $\begin{array}{l}\text { H-MoSe } \\
\text { H-TaSSe: } 1 \times 1 \\
1 \times 1\end{array}$ & $\begin{array}{c}0 \\
-2.59 \\
\end{array}$ & $3.58 / 3.81$ \\
\hline $\mathrm{H}-\mathrm{MoSe}_{2} / \mathrm{H}-\mathrm{TiSSe} / \mathrm{H}-\mathrm{MoSe}_{2}$ & $\begin{array}{c}\mathrm{H}_{-\mathrm{MoSe}_{2}:} 1 \times 1 \\
\text { H-TiSSe: } 1 \times 1\end{array}$ & $\begin{array}{c}0 \\
-2.93 \\
\end{array}$ & $3.63 / 3.86$ \\
\hline H-MoSe $2 / \mathrm{H}-W \mathrm{SeTe} / \mathrm{H}-\mathrm{MoSe}_{2}$ & $\begin{array}{l}\mathrm{H}_{-\mathrm{MoSe}_{2}: 1 \times 1} \times 1 \\
\text { H-WSeTe: } 1 \times 1\end{array}$ & $\begin{array}{c}0 \\
-3.29\end{array}$ & $3.66 / 3.58$ \\
\hline $\mathrm{H}-\mathrm{MoS}_{2} / \mathrm{H}-\mathrm{WSSe} / \mathrm{H}-\mathrm{MoS}_{2}$ & $\begin{array}{l}\text { H-MoS }: 1 \times 1 \\
\text { H-WSSe: } 1 \times 1\end{array}$ & $\begin{array}{c}0 \\
-2.10\end{array}$ & $3.54 / 3.65$ \\
\hline H-MoSe $2 / \mathrm{H}-\mathrm{WSTe} / \mathrm{H}-\mathrm{MoSe}_{2}$ & $\begin{array}{l}\text { H-MoSe }_{2}: 1 \times 1 \\
\text { H-WSTe: } 1 \times 1\end{array}$ & $\begin{array}{c}0 \\
-1.25 \\
\end{array}$ & $3.64 / 3.68$ \\
\hline $\mathrm{H}-\mathrm{MoSe}_{2} / \mathrm{H}-\mathrm{ZrBrCl} / \mathrm{H}-\mathrm{MoSe}_{2}$ & $\begin{array}{l}\mathrm{H}-\mathrm{MoSe}_{2}: 1 \times 1 \\
\mathrm{H}-\mathrm{ZrBrCl}: 1 \times 1 \\
\end{array}$ & $\begin{array}{c}0 \\
-4.82 \\
\end{array}$ & $3.69 / 3.57$ \\
\hline $\mathrm{H}-\mathrm{MoSe}_{2} / \mathrm{H}-\mathrm{TiBrCl} / \mathrm{H}-\mathrm{MoSe}_{2}$ & $\begin{array}{l}\mathrm{H}-\mathrm{MoSe}_{2}: 1 \times 1 \\
\mathrm{H}-\mathrm{TiBrCl}: 1 \times 1\end{array}$ & $\begin{array}{c}0 \\
-1.75\end{array}$ & $3.78 / 3.64$ \\
\hline
\end{tabular}

shown.

Another obvious application is to use Janus monolayers to lower the SB at metal/SC interfaces. For a general metal/SC interface, where the Fermi level of the metal is located between the valence band maximum (VBM) and conduction band minimum (CBM) of the $\mathrm{SC}$, the bands originating from the SC will bend at the interface creating a Schottky barrier. Below we show that by inserting a Janus monolayer between the metal and the SC one can 


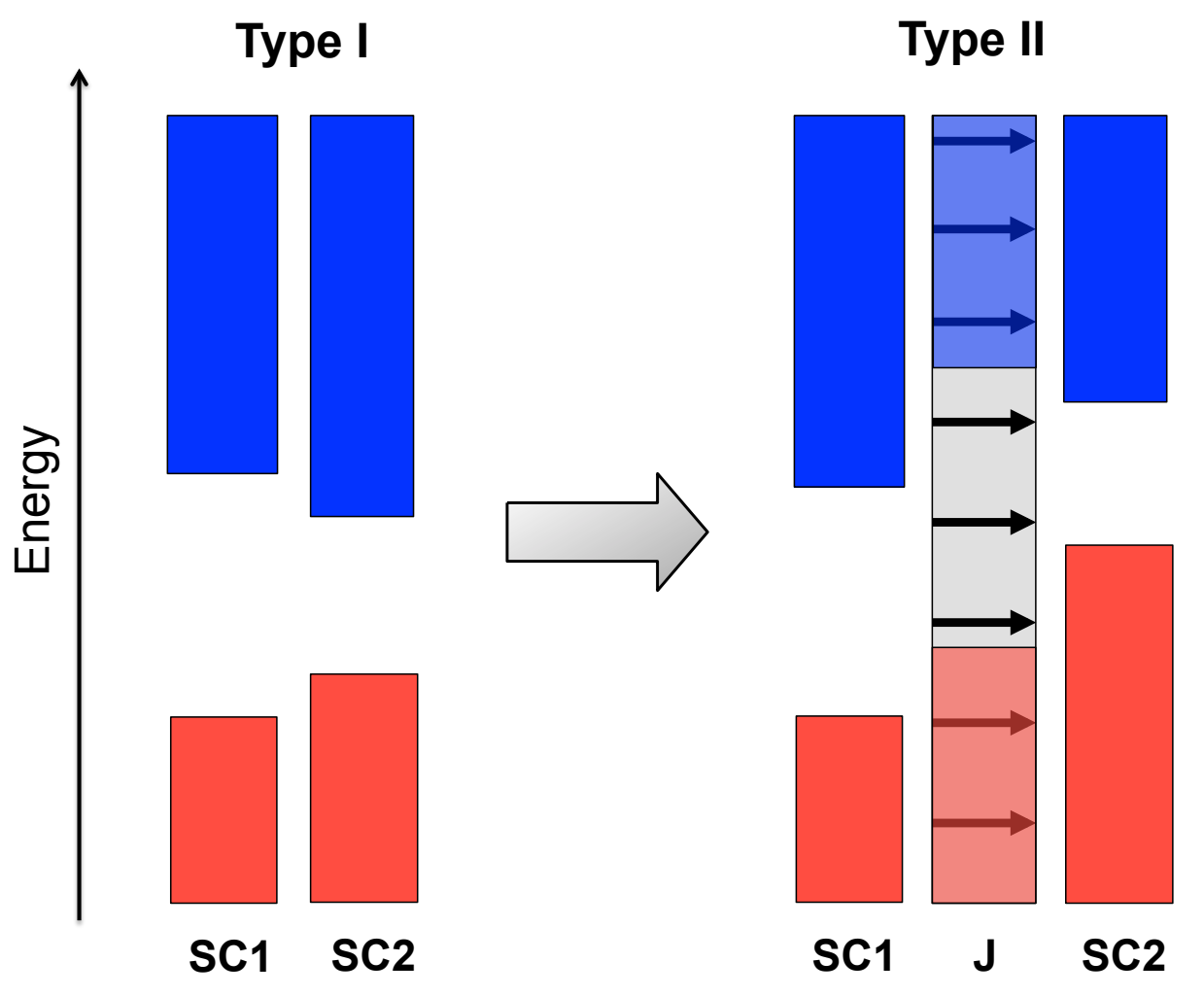

Figure 1: Left: sketch of a type I band alignment between two different semi-conductors. Right: sketch of the achieved type II band alignment between the two semi-conductors by sandwiching a Janus monolayer between them, which shifts the electrostatic potential on either side of the Janus monolayer. In a type III band alignment, the valence band maximum of one of the structures is located above the conduction band minimum of the other structure.

control the SB.

\section{Schottky-barriers at metal/SC interfaces}

To illustrate the concept in practice, we have calculated the band structure of both SC/SC and metal/SC vdW heterostructures with and without a Janus monolayer sandwiched inbetween. First consider the two band structures shown in figure 3. On the left is shown the band structure of bilayer $\mathrm{T}-\mathrm{MoS}_{2} / \mathrm{H}-\mathrm{MoS}_{2}$. We note that $\mathrm{MoS}_{2}$ is well-known to be 


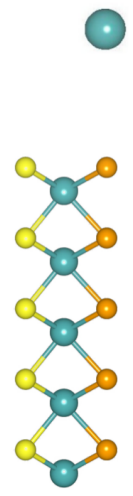

Mo
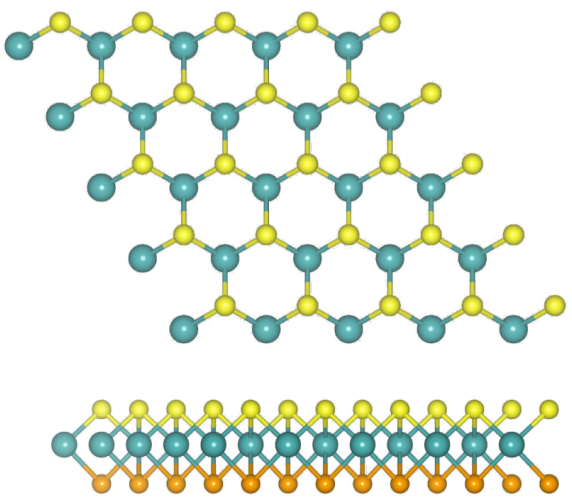

- Bi $\odot$ Te $\odot$ I
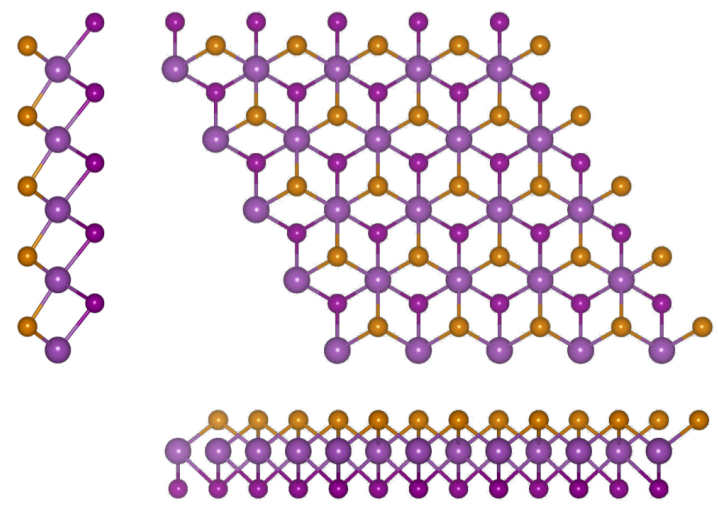

Figure 2: Top and side views of the atomic structures of the MoSSe prototype (left) and the BiTeI prototype (right).

metallic in the T-phase and semi-conducting in the H-phase. We note that the T-phase is in fact dynamically unstable and undergoes a transition to the T'-phase, however this is unimportant for the present discussion. On the right is shown the band structure of the same structure with H-MoSSe sandwiched in-between. For the two cases we consider an $\mathrm{AB}$ and $\mathrm{ABA}$ stacking respectively. The red colors represent the bands projected onto the T-MoS 2 layer, blue is projected onto the $\mathrm{H}-\mathrm{MoS}_{2}$ layer, and black is projected onto the Janus H-MoSSe layer. For the bilayer we see the bands from T-MoS 2 are crossing the Fermi level which overall renders the bilayer metallic, while the $\mathrm{H}-\mathrm{MoS}_{2}$ more or less preserves the size of its direct band gap at the K-point. The distance from the CBM of $\mathrm{H}-\mathrm{MoS}_{2}$ to the Fermi level is approximately $0.6 \mathrm{eV}$, which equals the $\mathrm{SB}$ of the interface. On the right, after insertion of the MoSSe layer, we see how the SB has essentially vanished. Now the CBM of the $\mathrm{H}-\mathrm{MoS}_{2}$ layer just touches the Fermi level, thus reducing the SB to almost zero. We note the $\mathrm{SB}$ of $\mathrm{T}-\mathrm{MoS}_{2} / \mathrm{H}-\mathrm{MoS}_{2}$ has previously been studied, where it was shown that the SB can be shifted $0.3 \mathrm{eV}$ by varying the stacking pattern. ${ }^{49}$ While this is an interesting result, the method proposed in our study gives the possibility to vary the SB, in a highly controlled manner, anywhere between the electronic band gap and essentially $0 \mathrm{eV}$, by varying the 
Janus monolayer material, which will be demonstrated later.
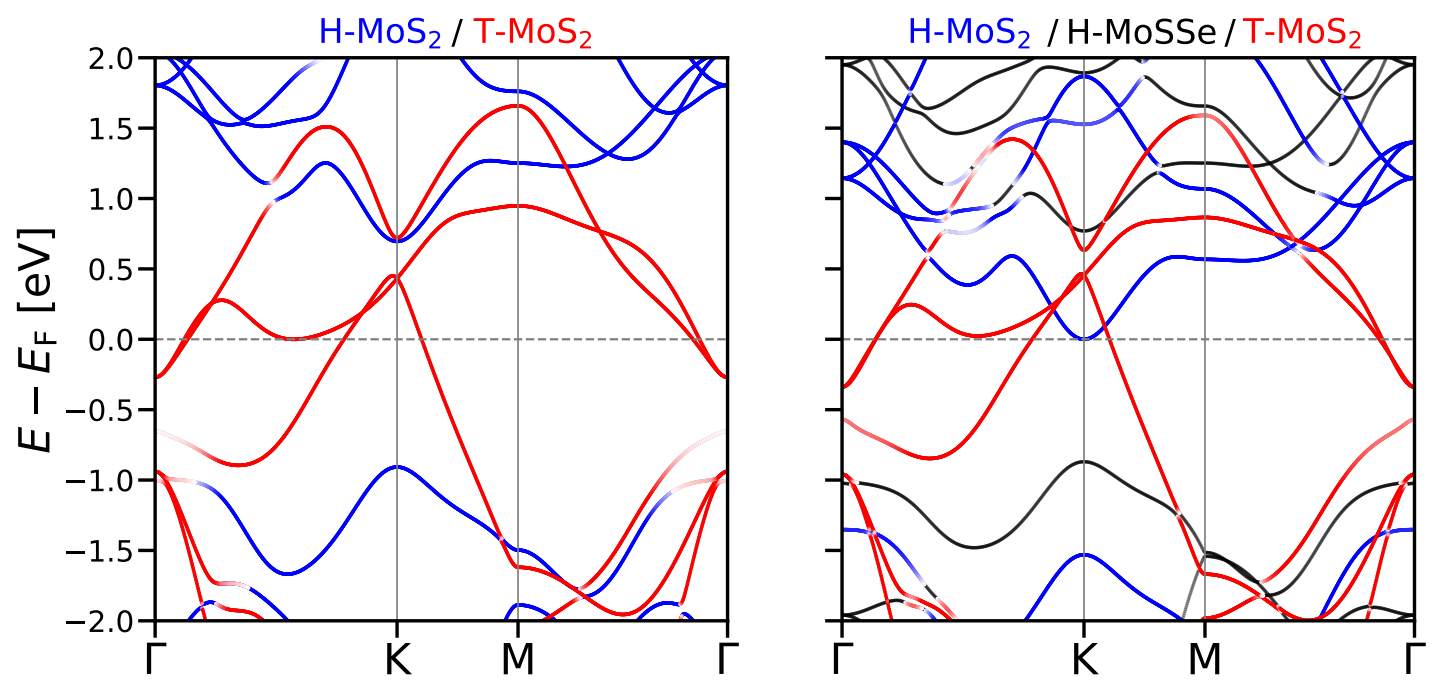

Figure 3: Left: Band structure of a bilayer consisting of $\mathrm{MoS}_{2}$ in the H- and T-phase respectively. Right: band structure of the same structure with the Janus monolayer MoSSe sandwiched in between the two layers. For both plots, red/blue is the projection of the bands onto the T- and H-phase of $\mathrm{MoS}_{2}$ respectively, while black is the projection onto MoSSe. Notice how the insertion of MoSSe shifts the bandedges of $\mathrm{H}-\mathrm{MoS}_{2}$ with respect to the Fermi level of the structure, effectively lowering the SB.

A more detailed picture of the electrostatic discontinuity induced by the Janus monolayer is shown in figure 4 . The top panel shows the electrostatic potential perpendicular to the T-MoS $/ \mathrm{H}_{2}-\mathrm{MoS}_{2}$ bilayer (averaged over the in-plane directions). The lower panel shows the same for the trilayer structure including H-MoSSe. Here the right hand side plot is a close-up of the upper part of the left plot. For the bilayer we see a minor change in the electrostatic potential across the bilayer of $0.03 \mathrm{eV}$. Since both T-MoS 2 and $\mathrm{H}-\mathrm{MoS}_{2}$ have a zero dipole moment in the out-of-plane direction in their freestanding monolayer form, this shift originates from a small interfacial dipole created at the interface. This phenomenon is well-known for metal-SC interfaces and has also been seen at graphene/SC interfaces. ${ }^{50}$ After insertion of H-MoSSe, we now find a shift of $0.63 \mathrm{eV}$. This large shift arises because of the internal dipole in the H-MoSSe layer, and gives the shift in the band structure we observed in figure 3. We here stress that we in this study consider the manipulation of SB defined 
by the SC band edges relative to the Fermi level and do not consider the tunnelling barrier evident from figure 4, that for some systems can impose an additional barrier. Modelling of such additional tunnelling barriers are system specific and will be the scope of future studies. It can be interesting to study how the intrinsic dipole moment of the Janus monolayers are affected by strain effects, since this is a widely used method in the world of 2D materials and van der Waals heterostructures to manipulate the properties of $2 \mathrm{D}$ monolayers. To do this, we calculate the vacuum level shift and band gap of strained monolayer H-MoSSe. The applied strain ranges from $-3 \%$ to $+3 \%$ (see fig. S1 in supporting information), which we believe are experimentally realizable values. We find that the vacuum level shift across monolayer MoSSe can be varied between $0.74 \mathrm{eV}$ to $0.77 \mathrm{eV}$. While this give rise to only little variation of the intrinsic dipole moment of the Janus monolayers, it shows that the proposed setup is very robust to strain effects. On the other hand the band gap changes over a range from $1.1 \mathrm{eV}$ to $1.6 \mathrm{eV}$ showing a great possible tunability of the electronic properties of monolayer Janus monolayers.

To further illustrate the concept, we consider the band structure of a bulk metal/SC interface and calculate the band structure of $\mathrm{H}-\mathrm{MoS}_{2}$ at a bulk $\mathrm{Au}(111)$ surface and at a bulk Pd(111) surface. The bulk metal surface is modelled by a slab of four atomic layers. The calculation has been performed in a $1 \times 1$ cell, with $\mathrm{H}-\mathrm{MoS}_{2}$ unstrained and $\mathrm{Au}$ and $\mathrm{Pd}$ strained significantly to match the lattice constant of $\mathrm{H}-\mathrm{MoS}_{2}$. Despite the very large strain, we find that the typical characteristics of both the $\mathrm{Au}$ and $\mathrm{Pd}$ band structures can be recognized, i.e. the wide $6 s p$ bands crossing the Fermi surface, and the flat $5 d$ bands at around $-1.5 \mathrm{eV}$ for $\mathrm{Au}$ and the $5 d$ bands crossing the Fermi level for Pd. This can be seen in figures 5 and 6 . Notice the same color coding has been used as for the previous band structure plot. Comparing the SB with and without H-MoSSe, we find a reduction of the SB of about $0.6 \mathrm{eV}$ for the $\mathrm{Au}$ surface, which is close to the reduction found for $\mathrm{T}-\mathrm{MoS}_{2} / \mathrm{H}-\mathrm{MoS} \mathrm{S}_{2}$. For the $\mathrm{Pd}$ surface the reduction is closer to $0.5 \mathrm{eV}$, showing a larger cancellation of the internal dipole of MoSSe in this case. We note here that is has been demonstrated that no chemical 

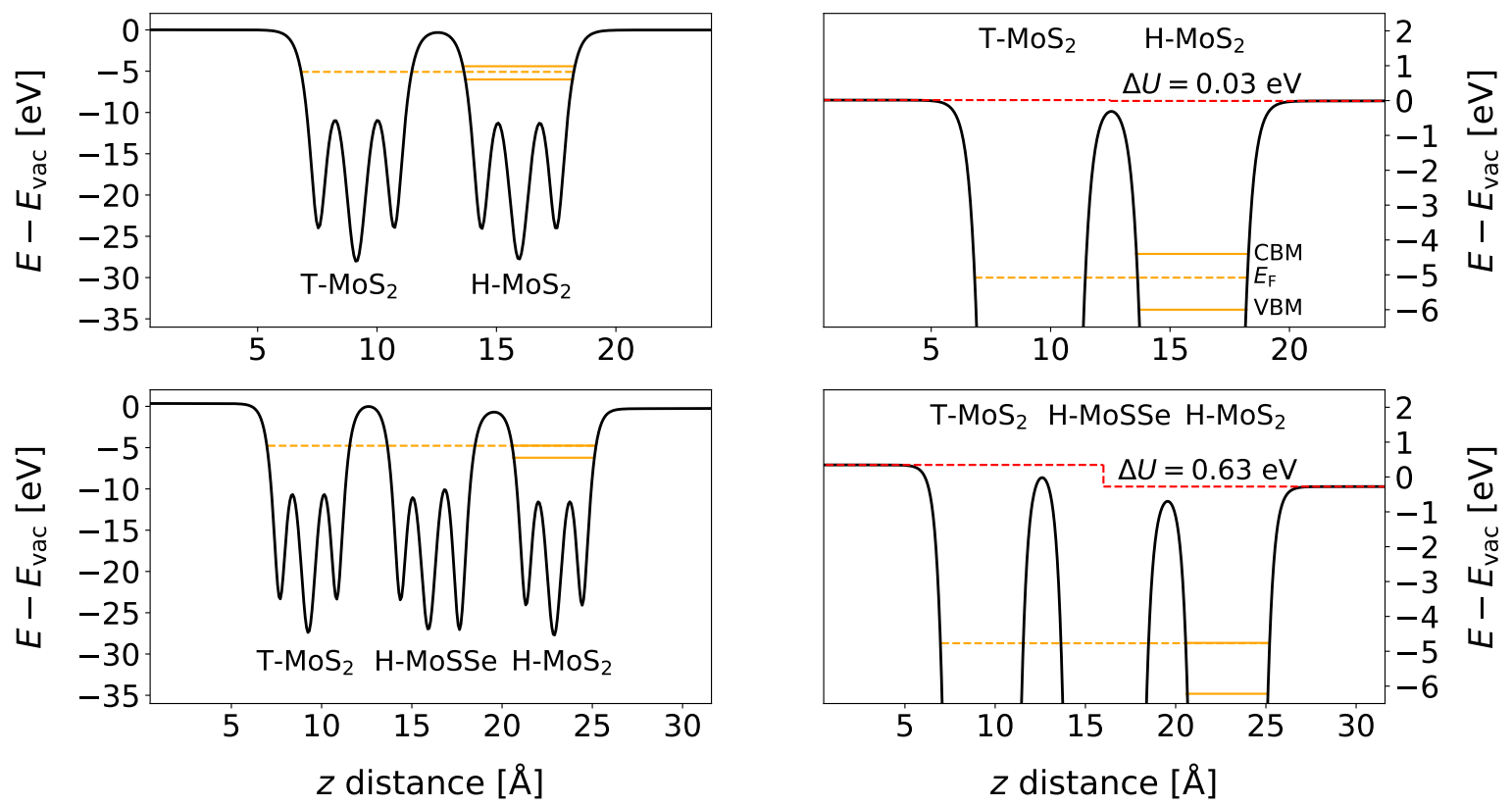

Figure 4: The electrostaic potential, with respect to the vacuum energy, for the bilayer and trilayer structures from figure 3 in the direction perpendicular to the layers. The right coloumn is a close-up of the top of the figures in the left coloumn showing the vacuum level shift $\Delta U$ on either side of the heterostructures. In orange is shown the VBM, CBM, and $E_{\mathrm{F}}$.

interaction are taking place at the $\mathrm{MoS}_{2} / \mathrm{Au}$ interface, while a considerable chemical bond strength is present at the $\mathrm{MoS}_{2} / \mathrm{Pd}$ interface. ${ }^{51}$ Thus, this stronger interaction explains the larger cancellation of the internal dipole. This is further stressed by the interlayer distance between $\mathrm{H}-\mathrm{MoS}_{2}$ and $\mathrm{Au} / \mathrm{Pd}$. For $\mathrm{H}-\mathrm{MoS}_{2} / \mathrm{Au}$ the smallest out-of-plane interlayer distance is $3.4 \AA$ while it is only $2.0 \AA$ for $\mathrm{H}-\mathrm{MoS}_{2} / \mathrm{Pd}$. This shows that the reduction of the outof-plane dipole moment is rather modest, even at metal surfaces that couple strongly to the Janus monolayer.

\section{Band alignment at SC/SC interfaces}

Next we consider how the band alignment can be tuned for SC/SC interfaces. Specifically, we consider bilayer $\mathrm{H}-\mathrm{MoS}_{2}$ with and without a H-MoSSe layer sandwiched in between. The band structure of both cases is depicted in figure 7 using the same colouring as in figure 

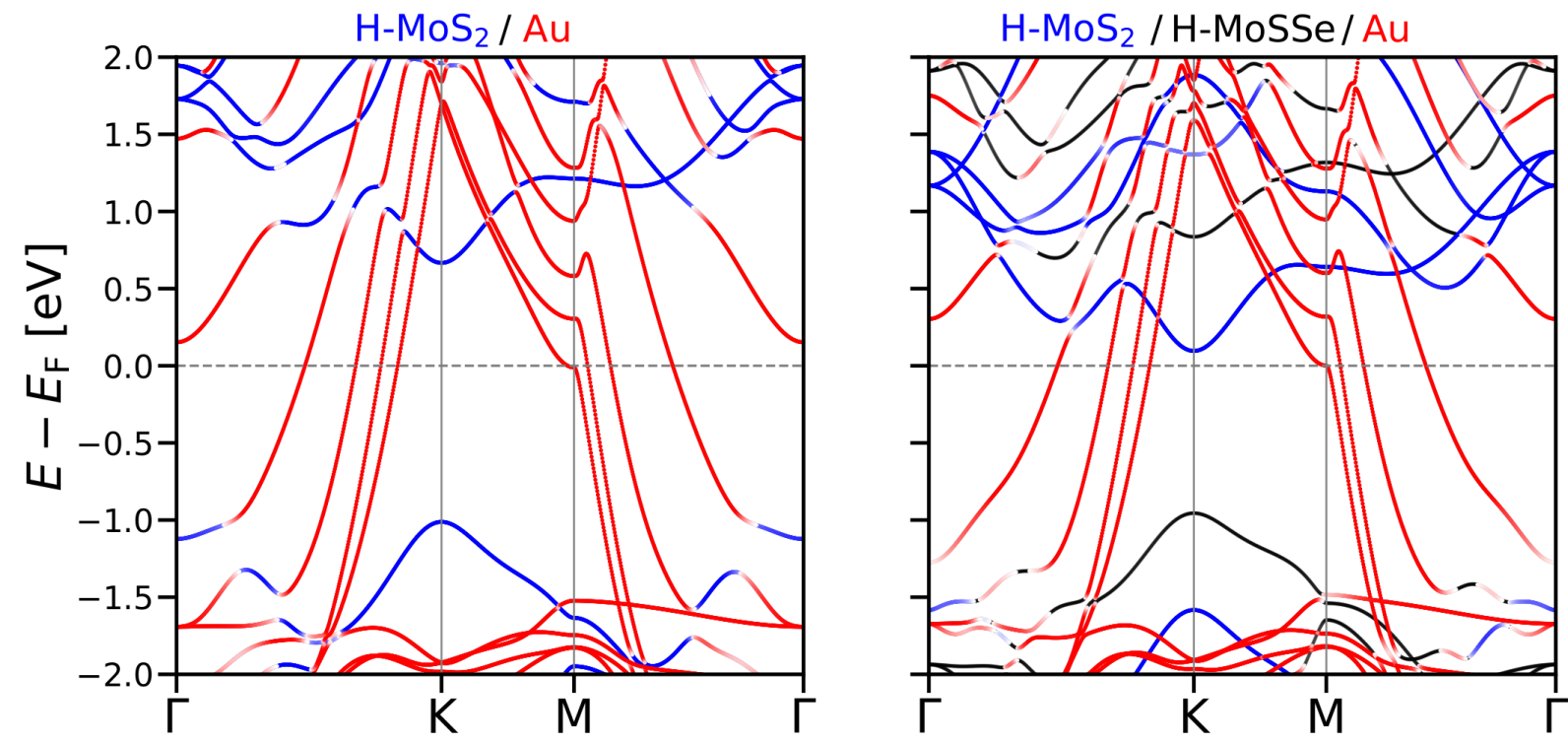

Figure 5: Left: Band structure of $\mathrm{H}-\mathrm{MoS}_{2}$ at a bulk Au metal surface. Right: band structure of the same structure with the Janus monolayer MoSSe sandwiched in between. For both plots, red/blue is the projection of the bands onto the $\mathrm{Au}$ and $\mathrm{H}-\mathrm{MoS}_{2}$ respectively, while black is the projection onto MoSSe.

3. We first notice the direct to in-direct band gap transition in going from monolayer to bilayer $\mathrm{H}-\mathrm{MoS}_{2}$, where the VBM is located at the K-point for monolayer $\mathrm{H}-\mathrm{MoS}_{2}$ and at the $\Gamma$-point for bilayer $\mathrm{H}-\mathrm{MoS}_{2}$. The two layers hybridize strongly around the $\Gamma$ point leading to a significant band splitting. In contrast, the interlayer hybridization is negligible around the K-point and the two bands are essentially degenerate. Therefore, the relative band positions at the K-point can be used to deduce the effect of electrostatic shift induced by the Janus monolayer. After insertion of the H-MoSSe layer we see the splitting of both the conduction and the valence bands at the K-point, effectively turning the perfect band alignment into a type-II band alignment. For the $\mathrm{H}-\mathrm{MoS}_{2} / \mathrm{H}-\mathrm{MoSSe} / \mathrm{H}-\mathrm{MoS}_{2}$ heterostructure we find a splitting of $0.72 \mathrm{eV}$, which equals that of the T- $\mathrm{MoS}_{2} / \mathrm{H}-\mathrm{MoSSe} / \mathrm{H}-\mathrm{MoS}_{2}$ heterostructure to within about $0.1 \mathrm{eV}$. Upon further stacking of Janus monolayers in a multilayer structure, recent studies ${ }^{48,52}$ have shown that the effect is to close the band gap, effectively creating a naturally doped p-n junction. This also shows that more than one Janus monolayer can be inserted if a larger shift in potential is desired. 

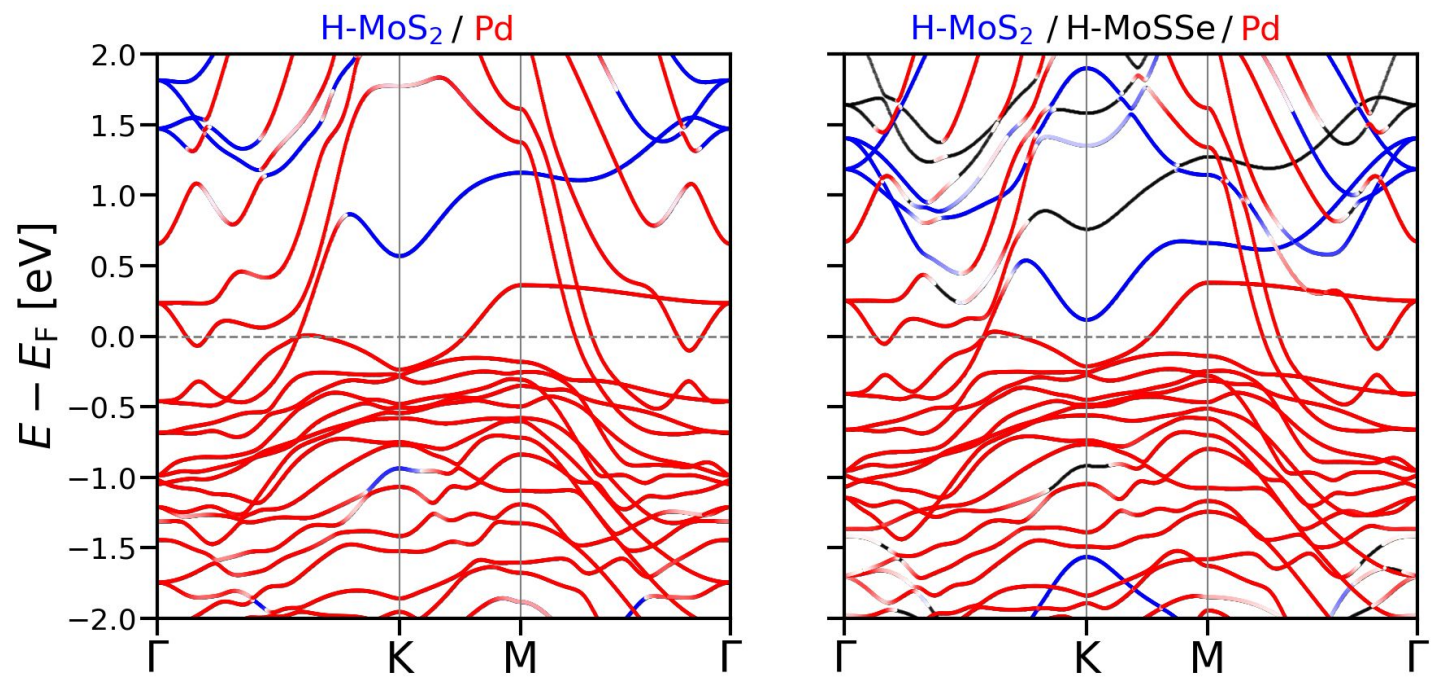

Figure 6: Same as figure 5 with a $\operatorname{Pd}(111)$ surface instead of an $\mathrm{Au}(111)$ surface.

It is also interesting to see how the interface can be controlled for a SC at a graphene monolayer because of the semi-metallic nature of graphene, and we therefore now consider the two graphene/hBN and graphene/H-MoSSe/hBN systems, where hBN is hexagonal boron nitride. In the same manner as for $\mathrm{T}-\mathrm{MoS}_{2} / \mathrm{H}-\mathrm{MoSSe} / \mathrm{H}-\mathrm{MoS}_{2}$, we find for graphene/HMoSSe/hBN that the bands of $\mathrm{hBN}$ shift down by 0.69 relative to the Fermi level after inclusion of H-MoSSe. This is larger than the shift observed for T-MoS $2 / \mathrm{H}-\mathrm{MoSSe} / \mathrm{H}-\mathrm{MoS}_{2}$, however the comparison to this system is somewhat cumbersome because in the latter case the conduction band gets pinned at the Fermi level. It is more interesting to observe that the shift is slightly smaller than for $\mathrm{H}-\mathrm{MoS}_{2} / \mathrm{H}-\mathrm{MoSSe} / \mathrm{H}-\mathrm{MoS}_{2}$. This observation can be explained by the larger cancellation of the internal dipole of H-MoSSe by graphene as compared to $\mathrm{H}-\mathrm{MoS}_{2}$. The larger cancellation of the internal dipole in H-MoSSe on graphene 

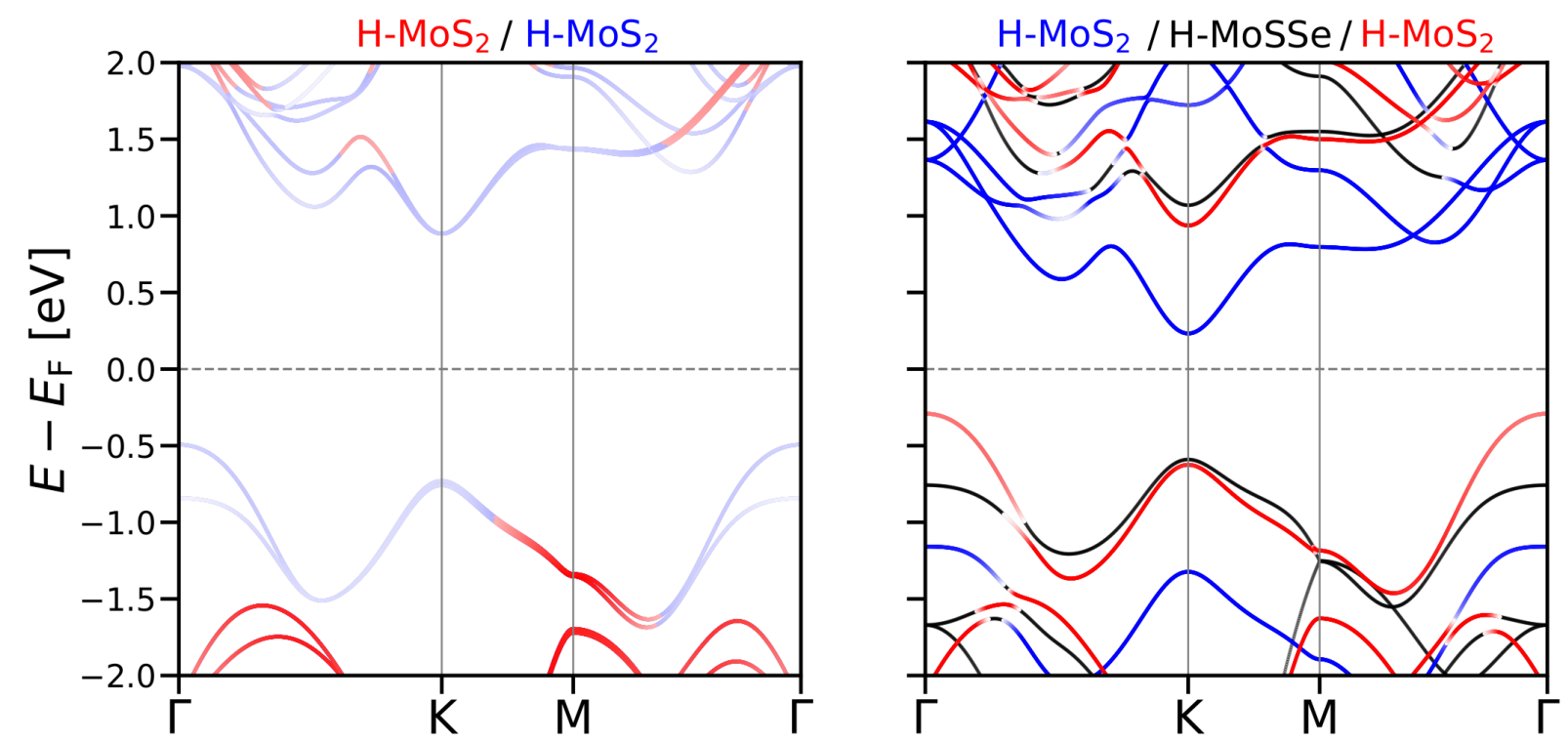

Figure 7: Left: Band structure of a bilayer $\mathrm{H}-\mathrm{MoS}_{2}$. Right: band structure of the same structure with the Janus monolayer MoSSe sandwiched in between the two layers. For both plots, red/blue is the projection of the bands onto the two $\mathrm{MoS}_{2}$ layers, while black is the projection onto MoSSe. Notice how the insertion of MoSSe splits the bands of the two $\mathrm{MoS}_{2}$ layers at the K-point.

compared to $\mathrm{H}-\mathrm{MoS}_{2}$, can be understood from the simple point that the $\mathrm{S}$ and Se atoms in H-MoSSe are spatially longer separated from the valence of $\mathrm{H}-\mathrm{MoS}_{2}$ (located on the Mo atoms) compared to the states in graphene. This together with the semi-metallic nature of graphene, a larger cancellation of the dipole on graphene is understandable.

To investigate whether the induced band splitting for a SC/Janus/SC vdWH can be predicted from the dipole moment of the freestanding Janus monolayer, we have calculated the band structure for 10 different SC/Janus/SC vdWHs. For a given trilayer the same SC is used on both sides (in analogy to the $\mathrm{H}-\mathrm{MoS}_{2} / \mathrm{H}-\mathrm{MoSSe} / \mathrm{H}-\mathrm{MoS}_{2}$ structure studied above). For the $\mathrm{SC}$ we use either $\mathrm{H}-\mathrm{MoS}_{2}$ or $\mathrm{H}-\mathrm{MoSe}_{2}$ (depending on what fits the lattice constant of the Janus monolayer better) and then investigate the effect of changing the Janus monolayer. The Janus monolayers have been chosen such that they span a wide range of dipole moments and their lattice constant are close to that of $\mathrm{MoS}_{2}$ or $\mathrm{MoSe}_{2}$ in order to minimize the applied strain (see the next section for a discussion of the other Janus monolayers). One exception is that we also include the structure $\mathrm{H}-\mathrm{WS}_{2} / \mathrm{H}-\mathrm{MoSSe} / \mathrm{H}-\mathrm{WS}_{2}$ to study the effect of the same 
Janus monolayer sandwiched between two different pairs of SCs. We use a in-plane $1 \times 1$ unit cell for the calculations, only straining the Janus monolayer to keep the band structure of the SC unchanged. We calculate the shift of the electrostatic potential on either side of the freestanding strained Janus monolayer, and the induced band shift of the SC in the $\mathrm{SC} /$ Janus/SC configuration. These values are plotted against each other in figure 8 . It is evident that the induced band shift of the bands between the two SCs in the $\mathrm{SC} / \mathrm{J} / \mathrm{SC}$ trilayer can be predicted from the out-of-plane dipole moment of the freestanding Janus monolayer. We note in passing that there is a linear relation between the shift in electrostatic potential and the internal out-of-plane dipole moment of the Janus monolayer. One more point are worth noticing from figure 8. First, we see to within $0.01 \mathrm{eV}$ the same band shift for the H$\mathrm{MoS}_{2} / \mathrm{MoSSe} / \mathrm{H}-\mathrm{MoS}_{2}$ and $\mathrm{H}-\mathrm{WS}_{2} / \mathrm{H}-\mathrm{MoSSe} / \mathrm{H}-\mathrm{WS}_{2}$, showing that the band shift induced by a Janus monolayer is only little affected by the choice of SC. This effect can be ascribed to the fact that the bands gets pinned at the Fermi level limiting the possible band shift (as seen above for $\mathrm{T}-\mathrm{MoS}_{2} / \mathrm{H}-\mathrm{MoSSe} / \mathrm{H}-\mathrm{MoS}_{2}$ ) and second, because for metallic heterostructures the free carriers can more effectively screen the internal dipole of the Janus monolayer. This is also the reason why the shift in potential is smaller in the SC/Janus/SC system compared to the potential shift for the freestanding Janus monolayer for all systems, since there will always be a small finite cancellation of the internal dipole moment. If the concentration of $\mathrm{S}$ and Se atoms on either side is altered this will affect the intrinsic out-of-plane dipole moment. To achieve the interface manipulations discussed in this chapter experimentally, we stress that the proposed method is highly dependent on the quality of the transfer of Janus monolayer into (or onto) the desired structures. 


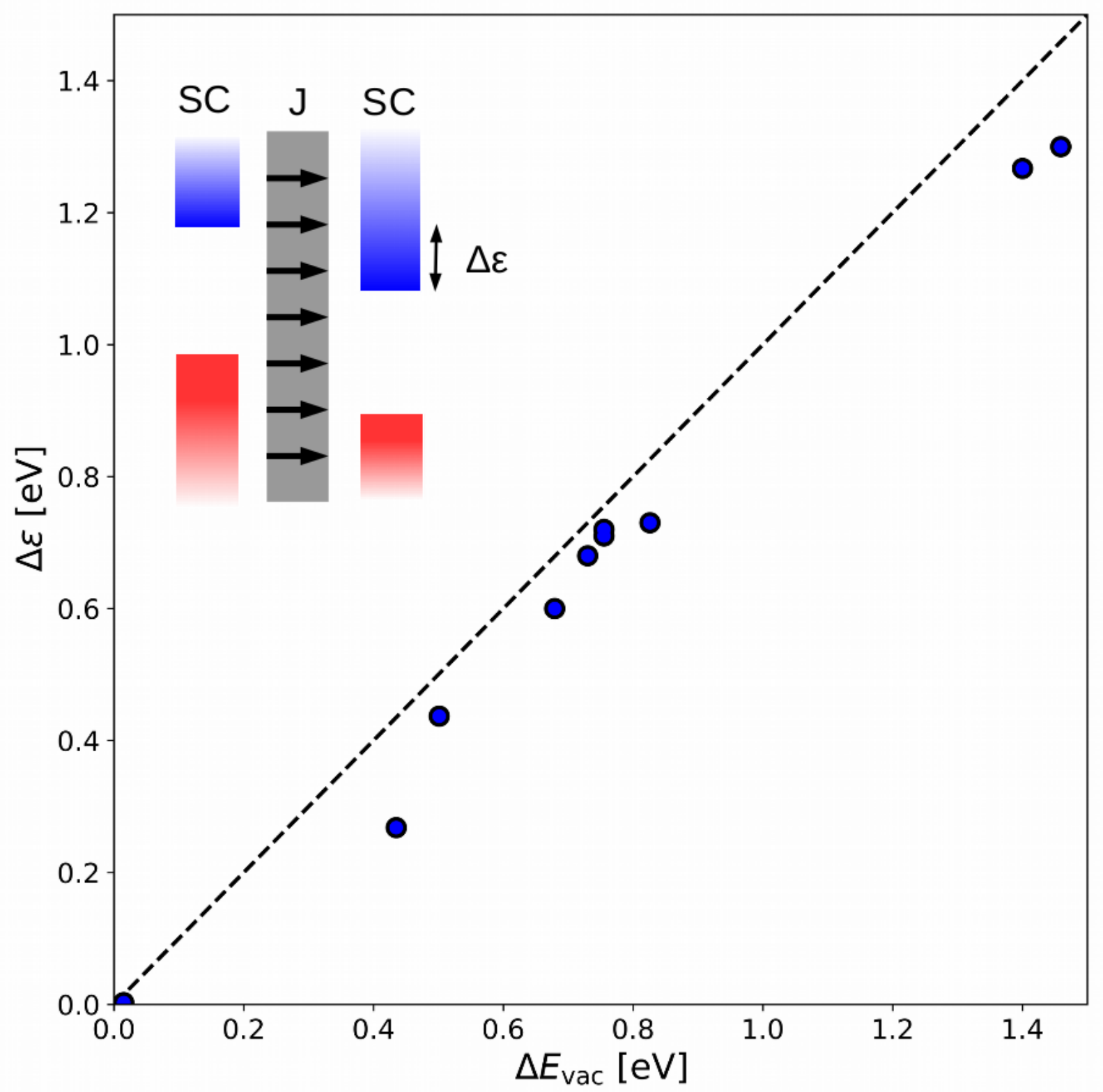

Figure 8: Splitting of the conduction bands $\Delta \epsilon$ for a SC bilayer, upon insertion of a Janus monolayer, as a function of the shift in potential across the freestanding Janus monolayer $\Delta E_{\text {vac }}$. Each data point represents a different Janus monolayer sandwiched between two identical SCs. From left to right the Janus monolayers are: TiSSe, $\mathrm{ZrBrCl}, \mathrm{TiBrCl}, \mathrm{WSeTe}$, WSSe, MoSSe, MoSSe, CrSSe, WSte, and MoSTe. The exact trilayer composition can then be deducted from table (1). We stress that there is little-to-no difference between trilayer $\mathrm{H}-\mathrm{MoS}_{2} / \mathrm{H}-\mathrm{MoSSe} / \mathrm{H}-\mathrm{MoS}_{2}$ and $\mathrm{H}-\mathrm{WS}_{2} / \mathrm{H}-\mathrm{MoSSe} / \mathrm{H}-\mathrm{WS}_{2}$. The fact that the shift in band energies are very close to the shift of the freestanding Janus monolayer, shows the highly predictive nature of the proposed strategy for band alignment engineering. 


\section{Janus Monolayer Library}

To further expand the prospects of the Janus monolayer controlled band alignment, we consider the Janus monolayers from our previous study, Riis-Jensen et al. ${ }^{53}$ In this study an initial set of 216 Janus monolayers with the chemical formula MXY in the H- and T-phases commonly known from the transition metal dichalcogenides (TMDs) were investigated.

In the following we adopt the notation used in the Computational 2D Materials Database $(\mathrm{C} 2 \mathrm{DB})^{54}$ nad in Riis-Jensen et al and refer to the $\mathrm{H}$ and $\mathrm{T}$ crystal structures as the MoSSe and BiTeI prototypes. All structures and properties shown in this work are available in the C2DB. The 216 candidate materials were constructed by combinatorial lattice decoration of the MoSSe and BiTeI prototype structures using elements with similar chemical properties. Specifically, for both MoSSe and BiTeI prototypes all possible combinations using one of the transition metals from group V, VI, and VII (for the central metal atom in the prototypes) in combination with two elements from either the pnictogens (As, $\mathrm{Sb}, \mathrm{Bi}$ ), the chalcogens $(\mathrm{S}, \mathrm{Se}, \mathrm{Te})$, or the halogens $(\mathrm{Cl}, \mathrm{Br}, \mathrm{I})$ were considered. In addition the study considered all combinations using one of the pnictogens as the metal atom and one element from the chalcogens and one element from the halogens. This makes a total of 108 candidate structures for each prototype. To assess the thermodynamic stability we calculate the heat of formation and the energy above the convex hull, with the latter defined as the most stable elementaries and binaries, see $\mathrm{C} 2 \mathrm{DB}^{44}$ for more details. For the dynamic stability we calculate the $\Gamma$-point phonons of the $2 \times 2$ cell as well as the elastic tensor. An imaginary phonon frequency or negative elastic constant implies a dynamically unstable material. In figure 9 we show the calculated energy above convex hull for all 216 structures. The MoSSe prototype is shown in blue and the BiTeI prototype is shown in orange. Taking uncertainties in the calculated heat of formation into account, we consider a material to be thermodynamically stable if its energy above convex hull is less than $0.1 \mathrm{eV} /$ atom as marked by the gray area. Points that have a black circle indicate materials that are dynamically stable and have a finite band gap.

We predict 47 materials that are both semi-conducting, thermodynamically stable, and 

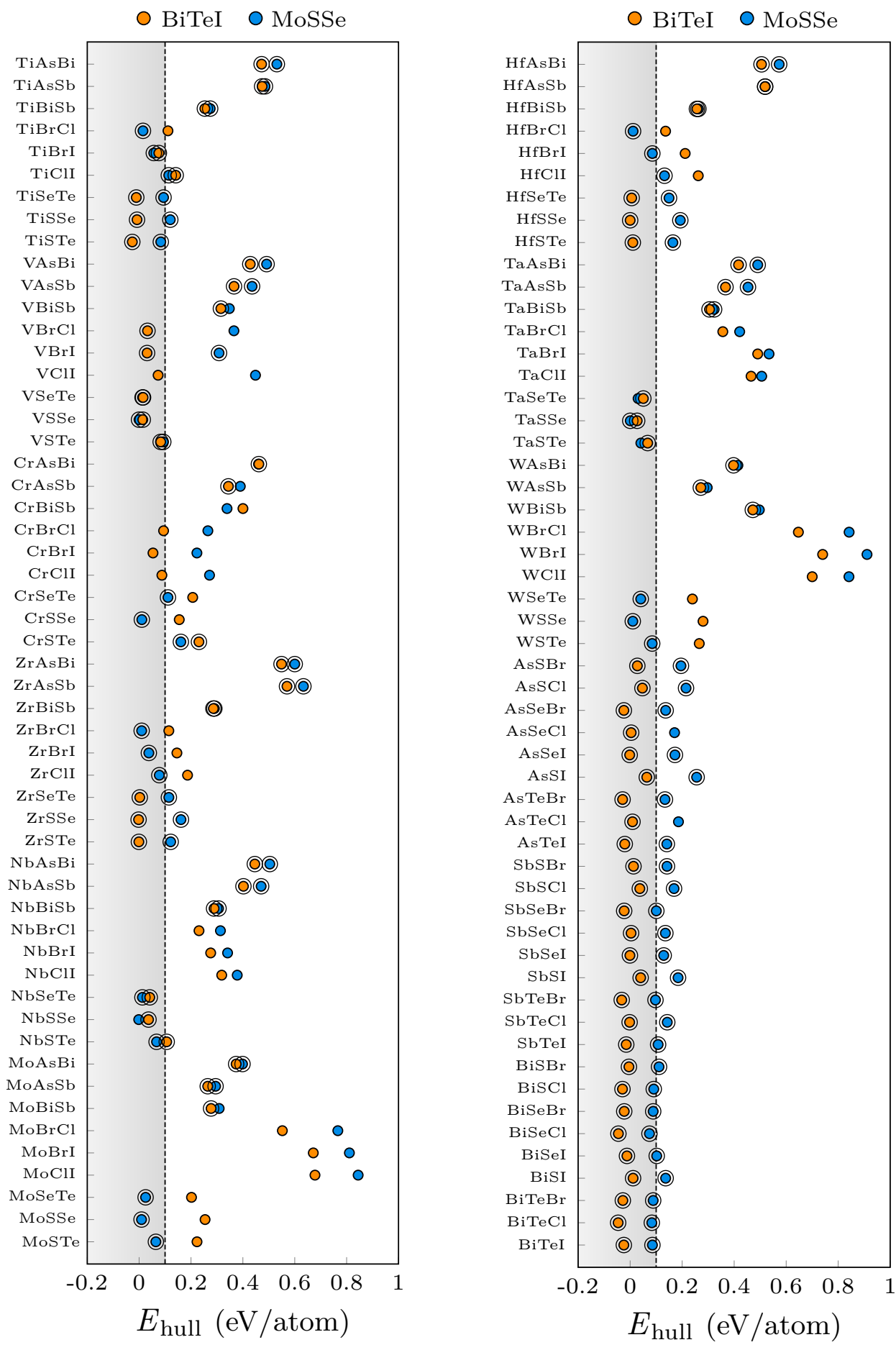

Figure 9: Energy above the convex hull for all Janus monolayers calculated in this study coloured according to their phase. Structures with an energy above convex hull lower than $0.1 \mathrm{eV} /$ atom are considered thermodynamically stable. Dynamical stability is shown by a black circle. 
dynamically stable. Out of these, 27 are in the BiTeI phase with a pnictogen atom as the central metal atom, and among these is the experimentally realized BiTeI. For the 47 materials we find to be semi-conducting and predict to be stable, we plot the shift in electrostatic potential across the material (red), together with the band edges (white/green boundaries) in figure 10. All stable semi-conducting materials, their in-plane lattice constant, electronic band gap, band gap center, the shift in the potential, heat of formation, and the energy above convex hull are summarized in table 2. The large variation in the band edge position and dipole strength underlines the flexibility of the proposed concept. 


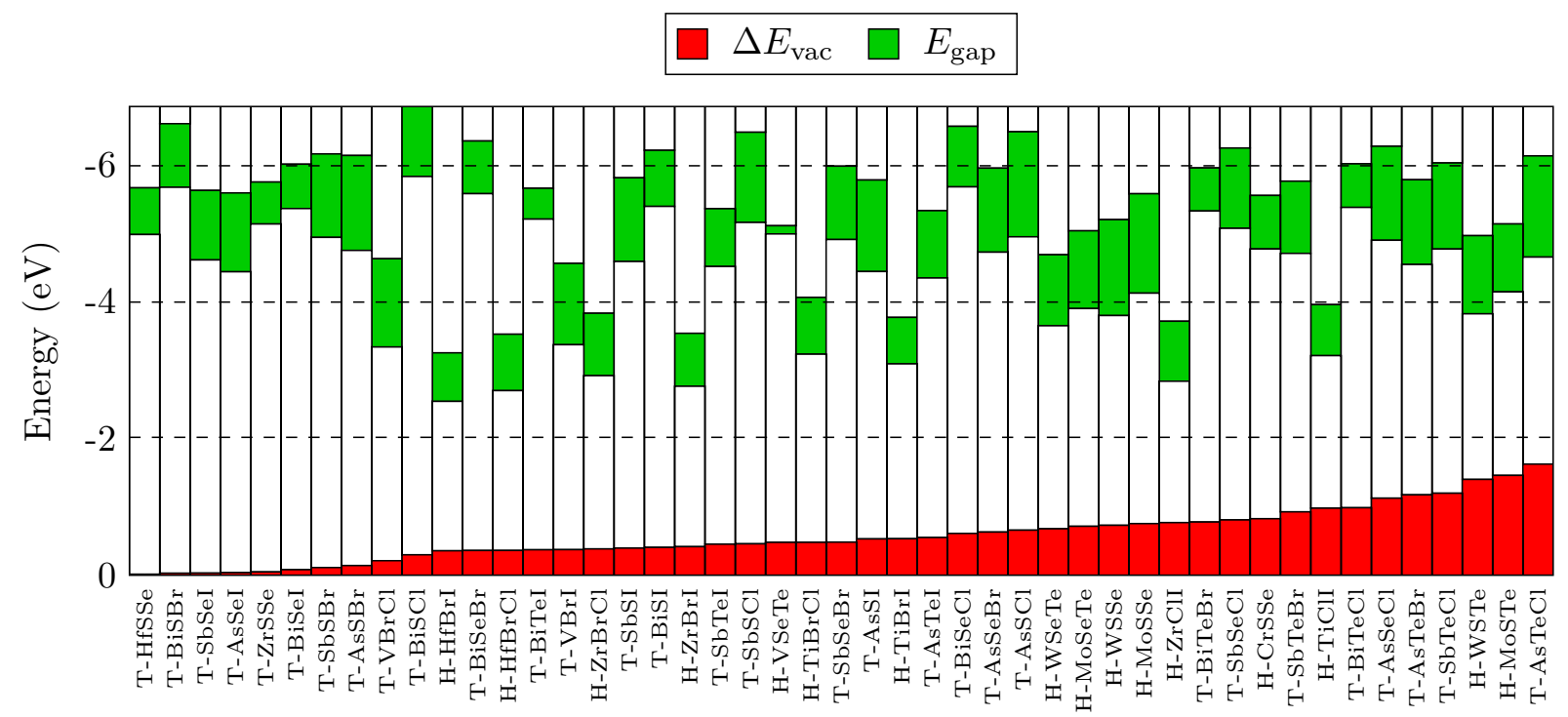

Figure 10: Overview of all stable Janus monolayers in the MoSSe (H-phase) and BiTeI (Tphase) prototype, sorted according to the vacuum level shift (red). The band gap is shown in green on an absolute scale relative to vacuum, such that the valence band maximum and conduction band minimum can be found from the white/green boundaries. All energies refer to PBE calculations. 
Table 2: Material properties of all semi-conducting stable materials candidates. The table contains information about: Elements, structural phase, in-plane lattice constant $[\AA], \mathrm{PBE}$ and HSE electronic band gap $[\mathrm{eV}]$, shift in electrostatic potential across the Janus monolayer $[\mathrm{eV}]$, the $\mathrm{PBE}$ band gap center relative to vacuum $[\mathrm{eV}]$, the heat of formation $[\mathrm{eV} /$ atom], and the energy above convex hull $[\mathrm{eV}]$.

\begin{tabular}{|c|c|c|c|c|c|c|c|c|}
\hline Material & Phase & $a(\AA)$ & $E_{\text {gap }}^{(\mathrm{PBE})}$ & $E_{\text {gap }}^{(\text {HSE })}$ & $\Delta E_{\mathrm{vac}}$ & $E_{\text {center }}^{(\mathrm{PBE})}$ & $\Delta H_{\mathrm{f}}$ & $\Delta H_{\text {hul }}$ \\
\hline HfSSe & $\mathrm{T}$ & 3.71 & 0.68 & 1.51 & 0.01 & 5.32 & -1.47 & -0.01 \\
\hline $\mathrm{BiSBr}$ & $\mathrm{T}$ & 4.11 & 0.93 & 1.60 & 0.03 & 6.13 & -0.56 & -0.00 \\
\hline SbSeI & $\mathrm{T}$ & 4.17 & 1.02 & 1.53 & 0.03 & 5.12 & -0.31 & -0.00 \\
\hline AsSeI & $\mathrm{T}$ & 3.93 & 1.15 & 1.68 & 0.04 & 5.01 & -0.16 & -0.00 \\
\hline $\mathrm{ZrSSe}$ & $\mathrm{T}$ & 3.74 & 0.61 & 1.51 & 0.05 & 5.44 & -1.48 & -0.01 \\
\hline BiSeI & $\mathrm{T}$ & 4.27 & 0.65 & 1.22 & 0.08 & 5.68 & -0.44 & -0.01 \\
\hline $\mathrm{SbSBr}$ & $\mathrm{T}$ & 3.99 & 1.22 & 1.84 & 0.11 & 5.55 & -0.42 & 0.01 \\
\hline AsSBr & $\mathrm{T}$ & 3.72 & 1.39 & 2.04 & 0.14 & 5.44 & -0.26 & 0.03 \\
\hline $\mathrm{VBrCl}$ & $\mathrm{T}$ & 3.75 & 1.29 & 3.77 & 0.21 & 3.98 & -1.01 & 0.00 \\
\hline $\mathrm{BiSCl}$ & $\mathrm{T}$ & 4.07 & 1.04 & 1.74 & 0.30 & 6.35 & -0.66 & -0.03 \\
\hline HfBrI & $\mathrm{H}$ & 3.64 & 0.71 & 1.16 & 0.36 & 2.90 & -0.89 & -0.00 \\
\hline $\mathrm{BiSeBr}$ & $\mathrm{T}$ & 4.19 & 0.77 & 1.36 & 0.36 & 5.96 & -0.58 & -0.02 \\
\hline $\mathrm{HfBrCl}$ & $\mathrm{H}$ & 3.43 & 0.82 & 1.36 & 0.36 & 3.11 & -1.34 & -0.08 \\
\hline BiTeI & $\mathrm{T}$ & 4.42 & 0.45 & 0.93 & 0.37 & 5.43 & -0.37 & -0.02 \\
\hline VBrI & $\mathrm{T}$ & 3.99 & 1.19 & 3.49 & 0.37 & 3.96 & -0.66 & 0.01 \\
\hline $\mathrm{ZrBrCl}$ & $\mathrm{H}$ & 3.49 & 0.91 & 1.40 & 0.38 & 3.37 & -1.51 & -0.02 \\
\hline SbSI & $\mathrm{T}$ & 4.08 & 1.22 & 1.78 & 0.40 & 5.20 & -0.28 & 0.04 \\
\hline BiSI & $\mathrm{T}$ & 4.19 & 0.82 & 1.47 & 0.41 & 5.80 & -0.41 & 0.01 \\
\hline $\mathrm{ZrBrI}$ & $\mathrm{H}$ & 3.70 & 0.77 & 1.17 & 0.42 & 3.15 & -1.09 & 0.02 \\
\hline SbTeI & $\mathrm{T}$ & 4.32 & 0.84 & 1.32 & 0.45 & 4.93 & -0.25 & -0.02 \\
\hline $\mathrm{SbSCl}$ & $\mathrm{T}$ & 3.94 & 1.32 & 1.99 & 0.46 & 5.82 & -0.51 & 0.04 \\
\hline VSeTe & $\mathrm{H}$ & 3.47 & 0.12 & 0.75 & 0.48 & 5.05 & -0.50 & 0.00 \\
\hline $\mathrm{TiBrCl}$ & $\mathrm{H}$ & 3.38 & 0.83 & 1.30 & 0.48 & 3.65 & -1.33 & -0.05 \\
\hline $\mathrm{SbSeBr}$ & $\mathrm{T}$ & 4.08 & 1.07 & 1.65 & 0.48 & 5.44 & -0.44 & -0.02 \\
\hline AsSI & $\mathrm{T}$ & 3.84 & 1.34 & 1.95 & 0.53 & 5.11 & -0.11 & 0.06 \\
\hline TiBrI & $\mathrm{H}$ & 3.63 & 0.68 & 1.04 & 0.53 & 3.43 & -0.88 & 0.04 \\
\hline AsTeI & $\mathrm{T}$ & 4.09 & 0.98 & 1.50 & 0.55 & 4.84 & -0.12 & -0.02 \\
\hline $\mathrm{BiSeCl}$ & $\mathrm{T}$ & 4.15 & 0.88 & 1.51 & 0.61 & 6.12 & -0.68 & -0.05 \\
\hline AsSeBr & $\mathrm{T}$ & 3.82 & 1.23 & 1.84 & 0.63 & 5.34 & -0.29 & -0.02 \\
\hline $\mathrm{AsSCl}$ & $\mathrm{T}$ & 3.65 & 1.54 & 2.26 & 0.66 & 5.71 & -0.35 & 0.05 \\
\hline WSeTe & $\mathrm{H}$ & 3.43 & 1.04 & 1.49 & 0.68 & 4.17 & -0.32 & 0.04 \\
\hline MoSeTe & $\mathrm{H}$ & 3.43 & 1.14 & 1.59 & 0.72 & 4.47 & -0.48 & 0.02 \\
\hline WSSe & $\mathrm{H}$ & 3.25 & 1.40 & 1.91 & 0.73 & 4.50 & -0.72 & 0.00 \\
\hline MoSSe & $\mathrm{H}$ & 3.25 & 1.45 & 1.95 & 0.75 & 4.85 & -0.81 & 0.00 \\
\hline $\mathrm{ZrClI}$ & $\mathrm{H}$ & 3.64 & 0.88 & 1.32 & 0.77 & 3.27 & -1.23 & 0.07 \\
\hline BiTeBr & $\mathrm{T}$ & 4.34 & 0.63 & 1.16 & 0.78 & 5.64 & -0.50 & -0.03 \\
\hline $\mathrm{SbSeCl}$ & $\mathrm{T}$ & 4.03 & 1.17 & 1.80 & 0.81 & 5.66 & -0.54 & 0.00 \\
\hline CrSSe & $\mathrm{H}$ & 3.13 & 0.78 & 1.24 & 0.83 & 5.16 & -0.57 & -0.00 \\
\hline $\mathrm{SbTeBr}$ & $\mathrm{T}$ & 4.24 & 1.06 & 1.62 & 0.93 & 5.23 & -0.38 & -0.03 \\
\hline TiClI & $\mathrm{H}$ & 3.55 & 0.75 & 1.19 & 0.98 & 3.58 & -1.01 & 0.07 \\
\hline $\mathrm{BiTeCl}$ & $\mathrm{T}$ & 4.30 & 0.64 & 1.20 & 0.99 & 5.69 & -0.60 & -0.05 \\
\hline $\mathrm{AsSeCl}$ & $\mathrm{T}$ & 3.76 & 1.37 & 2.06 & 1.13 & 5.58 & -0.38 & 0.00 \\
\hline AsTeBr & $\mathrm{T}$ & 3.98 & 1.24 & 1.84 & 1.18 & 5.16 & -0.24 & -0.03 \\
\hline $\mathrm{SbTeCl}$ & $\mathrm{T}$ & 4.19 & 1.26 & 1.87 & 1.20 & 5.40 & -0.47 & -0.00 \\
\hline WSTe & $\mathrm{H}$ & 3.36 & 1.14 & 1.68 & 1.40 & 4.39 & -0.42 & 0.08 \\
\hline MoSTe & $\mathrm{H}$ & 3.36 & 0.99 & 1.59 & 1.46 & 4.64 & -0.55 & 0.06 \\
\hline AsTeCl & $\mathrm{T}$ & 3.93 & 1.48 & 2.15 & 1.62 & 5.39 & -0.32 & 0.01 \\
\hline
\end{tabular}




\section{Conclusion}

We have proposed, and critically assessed, a new method for tuning the band line-up at solid state interfaces by insertion of a Janus monolayer in the interface. Due to its outof-plane dipole moment, the Janus monolayer creates a step in the electrostatic potential, which gives rise to a relative shift of the band energies on the two sides of the interface. Our DFT calculations show that the dipole of the Janus monolayer is almost unperturbed by the interface. Consequently, the shift in band alignment is determined by the intrinsic dipole of the Janus monolayer and therefore can be predicted to high accuracy. This important finding is a result of the inertness of the Janus monolayer and stands in contrast to existing methods, that involves formation of chemical bonds and complex charge transfer processes whose effects on the band energies are difficult to predict. Finally, we conducted a computational screening for new Janus monolayers and identified 47 (meta)stable candidates with a large range of out-of-plane dipole moments providing great flexibility for tuning of band alignment.

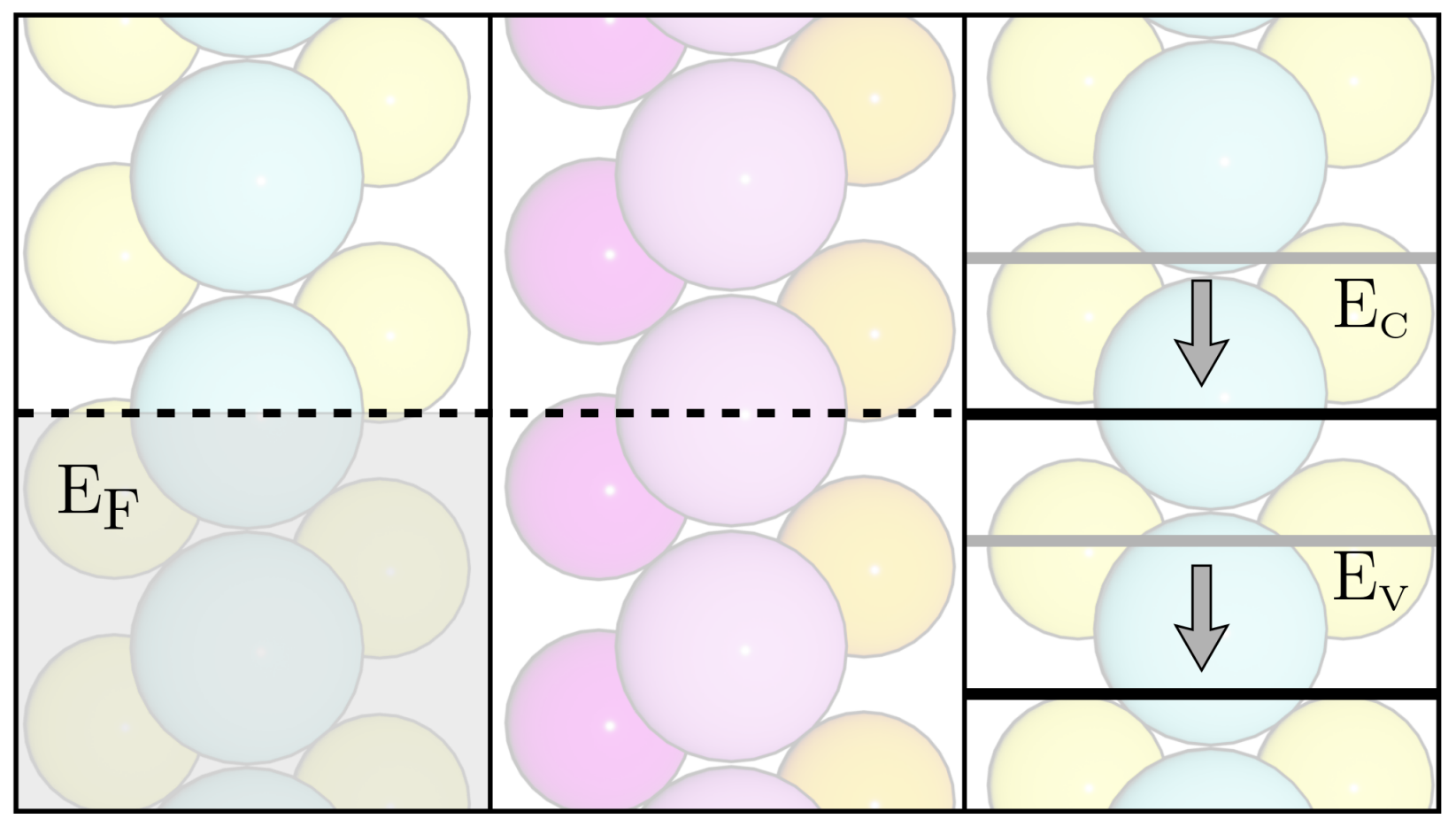

Figure 11: toc 


\section{Acknowledgement}

This project has received funding from the European Research Council (ERC) under the European Union's Horizon 2020 research and innovation programme (grant agreement No 773122, LIMA). The Center for Nanostructured Graphene is sponsored by the Danish National Research Foundation, Project DNRF103. 


\section{References}

(1) Novoselov, K. S.; Geim, A. K.; Morozov, S. V.; Jiang, D.; Zhang, Y.; Dubonos, S. V.; Grigorieva, I. V.; Firsov, A. A. Electric Field Effect in Atomically Thin Carbon Films. science 2004, 306, 666-669.

(2) Geim, A. K.; Grigorieva, I. V. Van der Waals Heterostructures. Nature 2013, 499, 419.

(3) Wu, S.; Buckley, S.; Schaibley, J. R.; Feng, L.; Yan, J.; Mandrus, D. G.; Hatami, F.; Yao, W.; Vučković, J.; Majumdar, A. et al. Monolayer Semiconductor Nanocavity Lasers with Ultralow Thresholds. Nature 2015, 520, 69.

(4) Ye, Y.; Wong, Z. J.; Lu, X.; Ni, X.; Zhu, H.; Chen, X.; Wang, Y.; Zhang, X. Monolayer Excitonic Laser. Nature Photonics 2015, 9, 733.

(5) Salehzadeh, O.; Djavid, M.; Tran, N. H.; Shih, I.; Mi, Z. Optically Pumped TwoDimensional MoS2 Lasers Operating at Room-Temperature. Nano letters 2015, 15, $5302-5306$.

(6) Linghu, J.; Yang, T.; Luo, Y.; Yang, M.; Zhou, J.; Shen, L.; Feng, Y. P. HighThroughput Computational Screening of Vertical 2D van der Waals Heterostructures for High-Efficiency Excitonic Solar Cells. ACS applied materials $\mathscr{G}$ interfaces 2018, 10, $32142-32150$.

(7) Bernardi, M.; Palummo, M.; Grossman, J. C. Semiconducting Monolayer Materials as a Tunable Platform for Excitonic Solar Cells. ACS nano 2012, 6, 10082-10089.

(8) Chhowalla, M.; Jena, D.; Zhang, H. Two-Dimensional Semiconductors for Transistors. Nature Reviews Materials 2016, 1, 16052.

(9) Tersoff, J. Schottky Barriers and Semiconductor Band Structures. Physical Review B 1985, 32, 6968. 
(10) Gong, C.; Colombo, L.; Wallace, R. M.; Cho, K. The Unusual Mechanism of Partial Fermi Level Pinning at Metal-MoS2 Interfaces. Nano Letters 2014, 14, 1714-1720, PMID: 24660782 .

(11) Henck, H.; Ben Aziza, Z.; Zill, O.; Pierucci, D.; Naylor, C. H.; Silly, M. G.; Gogneau, N.; Oehler, F.; Collin, S.; Brault, J. et al. Interface Dipole and Band Bending in the Hybrid $p-n$ Heterojunction $\mathrm{MoS}_{2} / \mathrm{GaN}(0001)$. Phys. Rev. B 2017, 96, 115312.

(12) Sahoo, A.; Nafday, D.; Paul, T.; Ruiter, R.; Roy, A.; Mostovoy, M.; Banerjee, T.; Saha-Dasgupta, T.; Ghosh, A. Out-of-Plane Interface Dipoles and Anti-Hysteresis in Graphene-Strontium Titanate Hybrid Transistor. npj 2D Materials and Applications 2018, 2, 1-7.

(13) Si, C.; Lin, Z.; Zhou, J.; Sun, Z. Controllable Schottky Barrier in GaSe/Graphene Heterostructure: the Role of Interface Dipole. 2D Materials 2016, 4, 015027.

(14) Kim, C.; Moon, I.; Lee, D.; Choi, M. S.; Ahmed, F.; Nam, S.; Cho, Y.; Shin, H.-J.; Park, S.; Yoo, W. J. Fermi Level Pinning at Electrical Metal Contacts of Monolayer Molybdenum Dichalcogenides. ACS Nano 2017, 11, 1588-1596, PMID: 28088846.

(15) Allain Adrien,; Kang Jiahao,; Banerjee Kaustav,; Kis Andras, Electrical Contacts to Two-Dimensional Semiconductors. Nature Materials 2015, 14, 1195.

(16) Das, S.; Chen, H.-Y.; Penumatcha, A. V.; Appenzeller, J. High Performance Multilayer MoS2 Transistors with Scandium Contacts. Nano Letters 2013, 13, 100-105, PMID: 23240655 .

(17) Kang, J.; Liu, W.; Banerjee, K. High-Performance MoS2 Transistors with LowResistance Molybdenum Contacts. Applied Physics Letters 2014, 104, 093106.

(18) Kang, J.; Liu, W.; Sarkar, D.; Jena, D.; Banerjee, K. Computational Study of Metal 
Contacts to Monolayer Transition-Metal Dichalcogenide Semiconductors. Phys. Rev. X 2014, 4, 031005.

(19) Guo, Y.; Liu, D.; Robertson, J. 3D Behavior of Schottky Barriers of 2D TransitionMetal Dichalcogenides. ACS Applied Materials \& Interfaces 2015, 7, 25709-25715, PMID: 26523332.

(20) Guo, Y.; Liu, D.; Robertson, J. Chalcogen Vacancies in Monolayer Transition Metal Dichalcogenides and Fermi Level Pinning at Contacts. Applied Physics Letters 2015, $106,173106$.

(21) Mönch, W. Role of Virtual Gap States and Defects in Metal-Semiconductor Contacts. Phys. Rev. Lett. 1987, 58, 1260-1263.

(22) Bokdam, M.; Brocks, G.; Katsnelson, M. I.; Kelly, P. J. Schottky Barriers at Hexagonal Boron Nitride/Metal Interfaces: A First-Principles Study. Physical Review B 2014, 90, 085415 .

(23) Anderson, R. Germanium-Gallium Arsenide Heterojunctions [letter to the editor]. IBM Journal of Research and Development 1960, 4, 283-287.

(24) Amani, M.; Chin, M.; Glen Birdwell, A.; P. O’Regan, T.; Najmaei, S.; Liu, Z.; M. Ajayan, P.; Lou, J.; Dubey, M. Electrical Performance of Monolayer MoS2 Field-Effect Transistors Prepared by Chemical Vapor Deposition. Applied Physics Letters 2013, 102.

(25) Wang Qing Hua,; Kalantar-Zadeh Kourosh,; Kis Andras,; Coleman Jonathan N.,; Strano Michael S., Electronics and Pptoelectronics of Two-Dimensional Transition Metal Dichalcogenides. Nature Nanotechnology 2012, 7, 699.

(26) Allain Adrien,; Kang Jiahao,; Banerjee Kaustav,; Kis Andras, Electrical Contacts to Two-Dimensional Semiconductors. Nature Materials 2015, 14, 1195. 
(27) Duan, X.; Wang, C.; Pan, A.; Yu, R.; Duan, X. Two-Dimensional Transition Metal Dichalcogenides as Atomically Thin Semiconductors: Opportunities and Challenges. Chem. Soc. Rev. 2015, 44, 8859-8876.

(28) Lembke, D.; Bertolazzi, S.; Kis, A. Single-Layer MoS2 Electronics. Accounts of Chemical Research 2015, 48, 100-110, PMID: 25555202.

(29) Chhowalla, M.; Jena, D.; Zhang, H. Two-dimensional Semiconductors for Transistors. Nature Reviews Materials 2016, 1, 16052.

(30) Manzeli, S.; Ovchinnikov, D.; Pasquier, D.; V. Yazyev, O.; Kis, A. 2D Transition Metal Dichalcogenides. Nature Reviews Materials 2017, 2.

(31) Ma, Y.; Kou, L.; Li, X.; Dai, Y.; Heine, T. Two-Dimensional Transition Metal Dichalcogenides with a Hexagonal Lattice: Room-Temperature Quantum Spin Hall Insulators. Phys. Rev. B 2016, 93, 035442.

(32) Kou, L.; Frauenheim, T.; Chen, C. Nanoscale Multilayer Transition-Metal Dichalcogenide Heterostructures: Band Gap Modulation by Interfacial Strain and Spontaneous Polarization. The Journal of Physical Chemistry Letters 2013, 4, 1730-1736, PMID: 26282986.

(33) Radisavljevic B.,; Radenovic A.,; Brivio J.,; Giacometti V.,; Kis A., Single-Layer MoS2 Transistors. Nature Nanotechnology 2011, 6, 147.

(34) Min, K.-A.; Park, J.; Wallace, R. M.; Cho, K.; Hong, S. Reduction of Fermi Level Pinning at $\mathrm{Au}-\mathrm{MoS} 2$ Interfaces by Atomic Passivation on Au Surface. 2D Materials 2017, 4, 015019.

(35) Farmanbar, M.; Brocks, G. Controlling the Schottky Barrier at MoS2/Metal Contacts by Inserting a BN Monolayer. Phys. Rev. B 2015, 91, 161304. 
(36) Chuang, S.; Battaglia, C.; Azcatl, A.; McDonnell, S.; Kang, J. S.; Yin, X.; Tosun, M.; Kapadia, R.; Fang, H.; Wallace, R. M. et al. MoS2 P-type Transistors and Diodes Enabled by High Work Function MoOx Contacts. Nano Letters 2014, 14, 1337-1342, PMID: 24568656.

(37) McDonnell, S.; Azcatl, A.; Addou, R.; Gong, C.; Battaglia, C.; Chuang, S.; Cho, K.; Javey, A.; Wallace, R. M. Hole Contacts on Transition Metal Dichalcogenides: Interface Chemistry and Band Alignments. ACS Nano 2014, 8, 6265-6272, PMID: 24797712.

(38) Chen, J.-R.; Odenthal, P. M.; Swartz, A. G.; Floyd, G. C.; Wen, H.; Luo, K. Y.; Kawakami, R. K. Control of Schottky Barriers in Single Layer MoS2 Transistors with Ferromagnetic Contacts. Nano Letters 2013, 13, 3106-3110, PMID: 23746085.

(39) Nam, S.-G.; Cho, Y.; Lee, M.-H.; Shin, K. W.; Kim, C.; Yang, K.; Jeong, M.; Shin, H.J.; Park, S. Barrier Height Control in Metal/Silicon Contacts with Atomically Thin MoS2 and WS2 Interfacial Layers. 2D Materials 2018, 5, 041004.

(40) Enkovaara, J.; Rostgaard, C.; Mortensen, J. J.; Chen, J.; Dułak, M.; Ferrighi, L.; Gavnholt, J.; Glinsvad, C.; Haikola, V.; Hansen, H. A. et al. Electronic Structure Calculations with GPAW: a Real-Space Implementation of the Projector AugmentedWave Method. Journal of Physics: Condensed Matter 2010, 22, 253202.

(41) Perdew, J. P.; Burke, K.; Ernzerhof, M. Generalized Gradient Approximation Made Simple. Phys. Rev. Lett. 1996, 77, 3865-3868.

(42) Monkhorst, H. J.; Pack, J. D. Special Points for Brillouin-Zone Integrations. Phys. Rev. $B$ 1976, 13, 5188-5192.

(43) Wellendorff, J.; Lundgaard, K. T.; Møgelhøj, A.; Petzold, V.; Landis, D. D.; Nørskov, J. K.; Bligaard, T.; Jacobsen, K. W. Density Functionals for Surface Science: Exchange-Correlation Model Development with Bayesian Error Estimation. Phys. Rev. B 2012, 85, 235149. 
(44) Haastrup, S.; Strange, M.; Pandey, M.; Deilmann, T.; Schmidt, P. S.; Hinsche, N. F.; Gjerding, M. N.; Torelli, D.; Larsen, P. M.; Riis-Jensen, A. C. et al. The Computational 2D Materials Database: high-throughput modeling and discovery of atomically thin crystals. 2D Materials 2018, 5, 042002.

(45) Zhang, J.; Jia, S.; Kholmanov, I.; Dong, L.; Er, D.; Chen, W.; Guo, H.; Jin, Z.; Shenoy, V. B.; Shi, L. et al. Janus Monolayer Transition-Metal Dichalcogenides. ACS Nano 2017, 11, 8192-8198, PMID: 28771310.

(46) Lu Ang-Yu,; Zhu Hanyu,; Xiao Jun,; Chuu Chih-Piao,; Han Yimo,; Chiu Ming-Hui,; Cheng Chia-Chin,; Yang Chih-Wen,; Wei Kung-Hwa,; Yang Yiming, et al. Janus Monolayers of Transition Metal Dichalcogenides. Nature Nanotechnology 2017, 12, 744.

(47) Fülöp, B.; Tajkov, Z.; Pető, J.; Kun, P.; Koltai, J.; Oroszlány, L.; Tóvári, E.; Murakawa, H.; Tokura, Y.; Bordács, S. et al. Exfoliation of Single Layer BiTeI Flakes. 2D Materials 2017,

(48) Riis-Jensen, A. C.; Pandey, M.; Thygesen, K. S. Efficient Charge Separation in 2D Janus van der Waals Structures with Built-in Electric Fields and Intrinsic p-n Doping. The Journal of Physical Chemistry C 2018, 122, 24520-24526.

(49) Hu, X.; Wang, Y.; Shen, X.; Krasheninnikov, A. V.; Sun, L.; Chen, Z. 1T phase as an Efficient Hole Injection Layer to TMDs Transistors: A Universal Approach to Achieve p-Type Contacts. 2D Materials 2018,

(50) Jin, C.; Rasmussen, F. A.; Thygesen, K. S. Tuning the Schottky Barrier at the Graphene/MoS2 Interface by Electron Doping: Density Functional Theory and ManyBody Calculations. The Journal of Physical Chemistry C 2015, 119, 19928-19933.

(51) Fontana Marcio,; Deppe Tristan,; Boyd Anthony K.,; Rinzan Mohamed,; Liu Amy Y.,; Paranjape Makarand,; Barbara Paola, Electron-Hole Transport and Photovoltaic Effect in Gated MoS2 Schottky Junctions. Scientific Reports 2013, 3, 1634. 
(52) Guan, Z.; Ni, S.; Hu, S. Tunable Electronic and Optical Properties of Monolayer and Multilayer Janus MoSSe as a Photocatalyst for Solar Water Splitting: A First-Principles Study. The Journal of Physical Chemistry C 2018, 122, 6209-6216.

(53) Riis-Jensen, A. C.; Deilmann, T.; Olsen, T.; Thygesen, K. S. Classifying the Electronic and Optical Properties of Janus Monolayers. ACS nano 2019, 13, 13354-13364.

(54) C2DB Webpage. https://cmr.fysik.dtu.dk/c2db/c2db.html, Accessed: 2019-0127. 